\title{
Modified Jelinski-Moranda Software Reliability Model with Imperfect Debugging Phenomenon
}

\author{
G. S. Mahapatra \\ Department of Engineering Sciences and \\ Humanities, \\ Siliguri Institute of Technology, \\ P.O.- Sukna, Siliguri-734009, West Bengal, India
}

\author{
P. Roy \\ Department of Engineering Sciences and \\ Humanities, \\ Siliguri Institute of Technology, \\ P.O.- Sukna, Siliguri-734009, West Bengal, India
}

\begin{abstract}
In this paper, we have modified the Jelinski-Moranda (J-M) model of software reliability using imperfect debugging process in fault removal activity. The J-M model was developed assuming the debugging process to be perfect which implies that there is one-to-one correspondence between the number of failures observed and faults removed. But in reality, it is possible that the fault which is supposed to have been removed may cause a new failure. In the proposed modified J-M model, we consider that whenever a failure occurs, the detected fault is not perfectly removed and there is a chance of raising new fault/faults due to wrong diagnosis or incorrect modifications in the software. In this paper, we develop a modified J-M model which can describe the imperfect debugging process. The parameters of our modified J-M model are estimated by using maximum-likelihood estimation method. Applicability of the model has been shown on the failure data set of Musa.
\end{abstract}

\section{Keywords}

Software reliability, Jelinski-Moranda model, Failure, Maximum likelihood estimation, Imperfect debugging.

\section{INTRODUCTION}

Over the last two decades, measurement of software reliability has become increasingly important because of rapid advancements in microprocessors and software. Today computer systems have been widely used for control of many complex systems. For critical systems failure of a computer system may result in disaster. The quality of software system can be described by many metrics such as complexity, portability, maintainability, availability, reliability, etc. Software reliability is a user oriented metric. The software failure is the departure of the software output from the system requirement and specification. There are many reasons for software to fail but usually these are attributed to the design problems resulting from new or changed requirements, revisions, corrections, etc. The software failures are introduced by the system analysts, designers, programmers and managers during different phases of the software development life cycle. To detect and remove these errors, the software system is tested. The quality of software system in terms of reliability is measured by the removal of these errors. Reliability is defined in terms of operational performance that one cannot measure before the product development is finished. In order to provide reliability indicators before the system is completely built, a reliability model is developed on the factors that affect reliability and the reliability predictions are made based on one's understanding of the system while it is under development $[1,2]$.
Software reliability is defined as the probability of failure-free operation of a computer program for a specified time in a specified environment [2]. Over the years, efforts made to estimate and measure software reliability has led to the development of many software reliability models. The J-M model [3] has a simple structure and assumptions. Use of the J-M model always yields an over optimistic reliability prediction [4]. Musa [5] proposed the basic execution time model with similar assumptions to the J-M model but introduced many important refinements. Goel and Okumoto [6] proposed the first Non Homogenous Poisson Process (NHPP) model. They assumed that the failure removal phenomenon follows NHPP. Yamada et al. [7] described the s-shapedness to the time delay between the failure observation and corresponding error removal. Ohba [8] attributed the sshapedness to the mutual dependency between the software errors. Most of the software reliability models assume that the error removal process (debugging) is perfect, i.e. when an attempt is made to remove a fault (cause of failure) the fault is removed with certainty. This assumption may be unrealistic, due to the complexity of software systems and vague understanding of the software requirements or specification. The testing team may not be able to remove the faults perfectly and the original error can be replaced by another error. The new fault may generate new failures when this part of the software system is traversed during the testing phase. The concept of imperfect debugging was first introduced in software reliability models by Goel [9] by introducing probability of imperfect debugging to the J-M model. Kapur and Garg [10] introduced the imperfect debugging process in the Goel and Okumoto model. They assumed that the error removal rate per remaining error is reduced due to the imperfect debugging. Chang and Liu [11] proposed a nonGaussian state space model to formulate an imperfect debugging phenomenon in software reliability. Shyur [12] developed the software reliability growth model with both imperfect debugging and change-point problem. Kapur et al. [13] presented a discrete software reliability growth model and the concept of two types of imperfect debugging during software fault removal phenomenon with Logistic Fault removal rate. Prasad et al. [14] used imperfect debugging and change-point problem into the software reliability growth model based on the well-known exponential distribution. Raju [15] discussed how to integrate a log-logistic testing-effort function into inflection s-shaped NHPP growth models to get a better description of the software fault detection phenomenon under imperfect debugging environment.

In this paper, we shall examine the J-M model, possibly the earliest and certainly one of the most well known black-box models. A modified J-M model is proposed in this paper assuming that the fault removal process is imperfect. The J-M 
model was developed assuming the debugging process to be perfect i.e. the detected fault is removed with certainty, this assumption is highly unrealistic. In reality, it is possible that the detected fault may not be removed perfectly and the fault, supposed to have been removed may cause a new failure. In our modified $\mathrm{J}-\mathrm{M}$ model, we consider that whenever a failure occurs, the detected fault is not perfectly removed and there is a chance of raising new fault/faults, due to wrong diagnosis or incorrect modifications in the software. We extend the J-M model by relaxing the assumptions of perfect debugging process and considering imperfect debugging process in fault removal activity. We consider that the probability of perfect debugging, the probability of imperfect debugging and the probability of raising new fault/s are independent of the testing time. We estimate the parameters of our modified J-M model using maximum likelihood estimation method. To check the validity of our modified J-M model, the model has been tested on the Musa system 1 failure data set. We have shown how the failure rate varies on failure number for the two models. Finally, the prediction analysis is presented and some conclusions are drawn.

The rest of the paper is organized as follows. Section 2 presents the classical J-M model, its assumptions and estimation of this model parameters. The modified J-M model with imperfect debugging phenomenon is presented in section 3 . This section discusses the assumptions, formulation and parameter estimation of the proposed model. Section 4 gives numerical results showing the data and prediction analysis of the J-M model and the modified J-M model using failure data set. Sensitivity analysis of the proposed model is also presented in this section. Finally, conclusions are drawn in section 5 .

\section{THE J-M MODEL}

The $\mathrm{J}-\mathrm{M}$ model $[1,3,16]$ is one of the earliest and most widely cited software reliability models to describe the failure behavior of a software system. It belongs to the exponential failure time class of models [1].

\subsection{Model Assumptions}

The assumptions made in the J-M model include the following:

(i) The number of initial software faults is unknown but fixed and constant.

(ii) Each fault in the software is independent and equally likely to cause a failure during a test.

(iii) Time intervals between occurrences of failure are independent, exponentially distributed random variables.

(iv) The software failure rate remains constant over the intervals between fault occurrences.

(v) The failure rate is proportional to the number of faults that remain in the software.

(vi) A detected fault is removed immediately and no new faults are introduced during the removal of the detected fault.

(vii) Whenever a failure occurs, the corresponding fault is removed with certainty.

\subsection{Model Formulation}

If the time between failure occurrences are $T_{i}=t_{i}-t_{i-1}, i=1,2, \ldots ., N$, then by the assumptions, the $T_{i}$ 's are exponentially distributed random variable with parameter $\lambda$ and mean is $1 / \lambda[1]$.

From the assumptions, the software failure rate at the $i^{\text {th }}$ failure interval i.e. the time between the $(i-1)^{\text {th }}$ and $i^{\text {th }}$ failure is given by
$\lambda\left(t_{i}\right)=\phi[N-(i-1)], \quad i=1,2, \ldots, N$

where

$\phi=$ a constant of proportionality denoting the failure rate contributed by each fault

$N=$ the initial number of faults in the software

$t_{i}=$ the time between $(i-1)^{\text {th }}$ and $i^{\text {th }}$ failure.

The failure density function and distribution function are as follows:

$$
\begin{aligned}
& f\left(t_{i}\right)=\phi[N-(i-1)] \exp \left(-\phi[N-(i-1)] t_{i}\right) \\
& \text { and } \\
& F_{i}\left(t_{i}\right)=1-\exp \left(-\phi[N-(i-1)] t_{i}\right)
\end{aligned}
$$

The reliability function at the $i^{\text {th }}$ failure interval is given by

$R\left(t_{i}\right)=1-F_{i}\left(t_{i}\right)=\exp \left(-\phi[N-(i-1)] t_{i}\right)$

and mean time to failure $(M T T F)$ for the $i^{\text {th }}$ fault $=$ $1 / \phi[N-(i-1)]$.

\subsection{Parameter Estimation}

If the failure data set $\left\{t_{1}, t_{2}, \ldots, t_{n} ; n>0\right\}$ is given, the parameters $N$ and $\phi$ in the J-M model can be estimated by using the maximum likelihood estimation method as follows:

$$
\hat{\phi}=\frac{n}{\hat{N}\left(\sum_{i=1}^{n} t_{i}\right)-\sum_{i=1}^{n}(i-1) t_{i}}
$$

and

$$
\sum_{i=1}^{n} \frac{1}{\hat{N}-(i-1)}=\frac{n}{\hat{N}-\left(\frac{1}{\sum_{i=1}^{n} t_{i}}\right)\left(\sum_{i=1}^{n}(i-1) t_{i}\right)}
$$

The maximum likelihood estimate of $\mathrm{N}$ i.e. $\hat{N}$ can be obtained by solving the equation (6). Substituting the estimated value of $\hat{N}$ from equation (6) into equation (5), we get the maximum likelihood estimate of $\phi$ i.e. $\hat{\phi}[1,16]$.

Then the current value of software reliability can be calculated by (4) as follows:

$$
R\left(t_{n+1}\right)=1-\hat{F}_{n+1}\left(t_{n+1}\right)=\exp \left(-\hat{\phi}(\hat{N}-n) t_{n+1}\right)
$$

\section{MODIFIED J-M MODEL WITH IMPERFECT DEBUGGING PHENOMENON}

The imperfect debugging is a common practical situation and the J-M model does not take this into account. The assumptions (vi) and (vii) of the J-M model state that whenever a failure occurs, the detected fault is removed with certainty and no new faults are inserted during the removal of the detected fault. These are highly unrealistic assumptions for the J-M model. We extend the J-M model by relaxing the assumptions of perfect debugging process and considering the imperfect debugging process in fault removal activity. During an imperfect debugging process, there can be two types of imperfect removal: (i) the fault is not removed successfully while no new faults are introduced and (ii) the fault is not removed successfully while new faults are created due to incorrect diagnoses. We consider the second type of imperfect removal and this type of debugging process is known as a birth-death Markov process [17]. This is the most practical situation in fault removing activity. We allow the imperfect debugging process to introduce new faults into the software due to incorrect modifications or diagnoses. 


\subsection{Model Assumptions}

The assumptions for our modified J-M model are similar to the J-M model except that it does not consider the perfect debugging process in fault removal activity. Our modified J$\mathrm{M}$ model assumes that the debugging process is truly imperfect. In order to modify the J-M model with imperfect debugging, we replace the assumptions (vi) and (vii) of the J$M$ model by the following new assumption:

Whenever a failure occurs, the detected fault is removed with probability $p$, the detected fault is not perfectly removed with probability $q$ and the new fault is generated with probability $r$. So it is obvious that $p+q+r=1$ and $q \geq r$.

\subsection{Model Formulation}

The software failure rate function between the $(i-1)^{\text {th }}$ and $i^{\text {th }}$ failure for our modified $\mathrm{J}-\mathrm{M}$ model with imperfect debugging is given by

$\lambda\left(t_{i}\right)=\phi[N-p(i-1)+r(i-1)]=\phi[N-(i-1)(p-r)]$

where $\phi, N$ and $t_{i}$ have the same meaning as defined in the J-M model.

The failure density and distribution functions are as follows

$f\left(t_{i}\right)=\phi[N-(i-1)(p-r)] \exp \left(-\phi[N-(i-1)(p-r)] t_{i}\right)$

and

$F_{i}\left(t_{i}\right)=1-\exp \left(-\phi[N-(i-1)(p-r)] t_{i}\right)$

The reliability function at the $i^{\text {th }}$ failure interval is given by

$R\left(t_{i}\right)=1-F_{i}\left(t_{i}\right)=\exp \left(-\phi[N-(i-1)(p-r)] t_{i}\right)$

and MTTF for the $i^{\text {th }}$ fault $=\frac{1}{\phi[N-(i-1)(p-r)]}$.

Note that if $p=1$ and $r=0$, then the failure behavior of the modified model becomes the same as the J-M model. Thus, the J-M model may be regarded as a special case of this modified model.

\subsection{Parameter Estimation}

Maximum likelihood estimation method has been used to estimate the parameters $N$ and $\phi$ of our modified J-M model. The parameters $N$ and $\phi$ are estimated as follows: Suppose that the failure data set $\left\{t_{1}, t_{2}, \ldots ., t_{n} ; n>0\right\}$ is given as in the J-M model. The likelihood function of the parameters $N$ and $\phi$ is given by

$$
\begin{aligned}
& L\left(t_{1}, t_{2}, \ldots, t_{n} ; \hat{N}, \hat{\phi}\right) \\
= & \prod_{i=1}^{n} f\left(t_{i}\right)=\prod_{i=1}^{n}\left[\hat{\phi}(\hat{N}-(i-1)(p-r)) \exp \left(-\hat{\phi}(\hat{N}-(i-1)(p-r)) t_{i}\right)\right] \\
= & \hat{\phi}^{n} \prod_{i=1}^{n}[\hat{N}-(i-1)(p-r)] \exp \left(-\hat{\phi} \sum_{i=1}^{n}[\hat{N}-(i-1)(p-r)] t_{i}\right)
\end{aligned}
$$

Taking the natural logarithm of the above likelihood function, we get

$$
\begin{aligned}
& \ln L \\
= & \ln \left[\hat{\phi}^{n} \prod_{i=1}^{n}[\hat{N}-(i-1)(p-r)] \exp \left(-\hat{\phi} \sum_{i=1}^{n}[\hat{N}-(i-1)(p-r)] t_{i}\right)\right] \\
= & n \ln \hat{\phi}+\sum_{i=1}^{n} \operatorname{In}\left[\hat{N}^{\prime}-(i-1)(p-r)\right] \\
& -\hat{\phi} \sum_{i=1}^{n}[\hat{N}-(i-1)(p-r)] t_{i}
\end{aligned}
$$

By taking the first partial derivative of the above loglikelihood function with respect to $\hat{N}$ and $\hat{\phi}$, respectively, and equating them to zero, we get the following likelihood equations:

$$
\begin{aligned}
& \frac{\partial}{\partial \hat{N}} \operatorname{In} L=\sum_{i=1}^{n} \frac{1}{[\hat{N}-(i-1)(p-r)]}-\hat{\phi} \sum_{i=1}^{n} t_{i}=0 \\
& \text { and } \\
& \frac{\partial}{\partial \hat{\phi}} \operatorname{In} L=\frac{n}{\hat{\phi}}-\sum_{i=1}^{n}[\hat{N}-(i-1)(p-r)] t_{i}=0
\end{aligned}
$$

From equation (15), we get

$$
\begin{aligned}
& \hat{\varphi}=\frac{n}{\sum_{i=1}^{n}[\hat{N}-(i-1)(p-r)] t_{i}}=\frac{n}{\sum_{i=1}^{n} \hat{N} t_{i}-\sum_{i=1}^{n}(i-1)(p-r) t_{i}} \\
& =\frac{n}{\hat{N} \sum_{i=1}^{n} t_{i}-\sum_{i=1}^{n}(i-1)(p-r) t_{i}}
\end{aligned}
$$

Now putting the value of $\hat{\phi}$ from equation (16) into equation (14), we obtain

$$
\sum_{i=1}^{n} \frac{1}{[\hat{N}-(i-1)(p-r)]}=\frac{n \sum_{i=1}^{n} t_{i}}{\hat{N} \sum_{i=1}^{n} t_{i}-\sum_{i=1}^{n}(i-1)(p-r) t_{i}}
$$

$$
\sum_{i=1}^{\text {or, }} \frac{1}{[\hat{N}-(i-1)(p-r)]}=\frac{n}{\hat{N}-\left(\frac{1}{\sum_{i=1}^{n} t_{i}}\right)\left(\sum_{i=1}^{n}(i-1)(p-r) t_{i}\right)}
$$

We get the maximum likelihood estimate $\hat{N}$ by solving the equation (17) and putting this estimated value into equation (16) to obtain the maximum likelihood estimate $\hat{\phi}$.

The software reliability function can be obtained from (11) as follows:

$$
R\left(t_{n+1}\right)=1-\hat{F}_{n+1}\left(t_{n+1}\right)=\exp \left(-\hat{\phi}[\hat{N}-n(p-r)] t_{n+1}\right)
$$

The estimated mean time to failure for the $(n+1)^{\text {th }}$ fault is

$$
M \hat{T T F}=\frac{1}{\hat{\phi}[\hat{N}-n(p-r)]} .
$$

\section{NUMERICAL EXAMPLE}

\subsection{Model validation using Musa Data Set}

In this section, we have concentrated on analysis of the software reliability data set published by Musa [18]. To check the validity of our modified J-M model, it is tested on the Musa system 1 failure data set. The values of $p$ and $r$ are supposed to be known. In all the existing software failure data sets, these values are not provided. The estimation of $p$ and $r$ from the failure data is also not possible since the parameters estimation tend to be unstable. Thus the values of $p$ and $r$ are assumed and for example, we consider as $p=0.93$ and $r=0.02$.

\subsubsection{Data analysis}

The data and estimates of parameters of both the J-M model and the modified J-M model with imperfect debugging are shown in Table 1. 
Table 1. Analysis of Musa system 1 failure data

\begin{tabular}{|c|c|c|c|c|c|c|c|c|c|c|c|}
\hline & & \multicolumn{5}{|c|}{ J-M Model } & \multicolumn{5}{|c|}{ Modified J-M Model } \\
\hline $\mathrm{N}$ & $t_{n}$ & $\hat{N}$ & $\hat{\phi}$ & $\hat{\lambda}$ & MTिTF & $\hat{F}_{n+1}\left(t_{n+1}\right)$ & $\hat{N}$ & $\hat{\phi}$ & $\hat{\lambda}$ & MTTF & $\hat{F}_{n+1}\left(t_{n+1}\right)$ \\
\hline 1 & 3 & 1 & 0.3333 & 0 & $\infty$ & 0 & 1 & 0.3333 & 0.0300 & 33.3333 & 0.5934 \\
\hline 2 & 30 & 2 & 0.0556 & 0 & $\infty$ & 0 & 2 & 0.0517 & 0.0093 & 107.5000 & 0.6505 \\
\hline 3 & 113 & 3 & 0.0165 & 0 & $\infty$ & 0 & 3 & 0.0146 & 0.0040 & 253.1358 & 0.2738 \\
\hline 4 & 81 & 4 & 0.0098 & 0 & $\infty$ & 0 & 4 & 0.0088 & 0.0032 & 315.2153 & 0.3057 \\
\hline 5 & \begin{tabular}{|l|}
115 \\
\end{tabular} & 6 & 0.0046 & 0.0046 & 218.6000 & 0.0403 & 5 & 0.0060 & 0.0027 & 372.1378 & 0.0239 \\
\hline 6 & 9 & 11 & 0.0021 & 0.0105 & 95.2333 & 0.0208 & 10 & 0.0023 & 0.0105 & 95.3142 & 0.0208 \\
\hline 7 & 2 & $\infty$ & 0 & 0.0198 & 50.4286 & 0.8354 & $\infty$ & 0 & 0.0198 & 50.4286 & 0.8354 \\
\hline 8 & 91 & 28 & 0.00074218 & 0.0148 & 67.3688 & 0.8103 & 25 & 0.00083370 & 0.0148 & 67.6903 & 0.8088 \\
\hline 9 & 112 & 16 & 0.0014 & 0.0099 & 100.7460 & 0.1383 & 15 & 0.0015 & 0.0102 & 98.2283 & 0.1416 \\
\hline 10 & 15 & 46 & 0.00042405 & 0.0153 & 65.5056 & 0.8784 & 42 & 0.00046426 & 0.0153 & 65.4698 & 0.8785 \\
\hline 11 & 138 & 20 & 0.0011 & 0.0098 & 102.1818 & 0.3870 & 18 & 0.0012 & 0.0097 & 103.1262 & 0.3842 \\
\hline 12 & 50 & 27 & 0.00075572 & 0.0113 & 88.2167 & 0.5822 & 25 & 0.00081211 & 0.0114 & 87.4542 & 0.5854 \\
\hline 13 & 77 & 29 & 0.00069496 & 0.0111 & 89.9327 & 0.2342 & 27 & 0.00074148 & 0.0112 & 88.9023 & 0.2366 \\
\hline 14 & 24 & 61 & 0.00030036 & 0.0141 & 70.8359 & 0.7823 & 56 & 0.00032682 & 0.0141 & 70.7293 & 0.7828 \\
\hline 15 & 108 & 39 & 0.00049358 & 0.0118 & 84.4167 & 0.6474 & 35 & 0.00055186 & 0.0118 & 84.8730 & 0.6454 \\
\hline 16 & 88 & 38 & 0.00050881 & 0.0112 & 89.3352 & 0.9994 & 35 & 0.00055060 & 0.0113 & 88.8557 & 0.9995 \\
\hline 17 & \begin{tabular}{|l|l|}
670 \\
\end{tabular} & 18 & 0.0014 & 0.0015 & 686.2353 & 0.1604 & 17 & 0.0015 & 0.0022 & 449.2957 & 0.2344 \\
\hline 18 & 120 & 20 & 0.0012 & 0.0023 & 429.9444 & 0.0587 & 18 & 0.0013 & 0.0021 & 470.3628 & 0.0538 \\
\hline 19 & 26 & 23 & 0.00089852 & 0.0036 & 278.2368 & 0.3362 & 21 & 0.00098070 & 0.0036 & 274.8461 & 0.3395 \\
\hline 20 & 114 & 25 & 0.00078204 & 0.0039 & 255.7400 & 0.7194 & 23 & 0.00084144 & 0.0040 & 247.5921 & 0.7309 \\
\hline 21 & \begin{tabular}{|l|}
325 \\
\end{tabular} & 24 & 0.00084378 & 0.0025 & 395.0476 & 0.1300 & 22 & 0.00091234 & 0.0026 & 379.2691 & 0.1350 \\
\hline 22 & 55 & 27 & 0.00068427 & 0.0034 & 292.2818 & 0.5631 & 25 & 0.00072668 & 0.0036 & 276.3307 & 0.5835 \\
\hline 23 & 242 & 28 & 0.00063944 & 0.0032 & 312.7739 & 0.1954 & 25 & 0.00073062 & 0.0030 & 336.2883 & 0.1831 \\
\hline 24 & 68 & 31 & 0.00054131 & 0.0038 & 263.9107 & 0.7979 & 28 & 0.00060325 & 0.0037 & 269.1065 & 0.7916 \\
\hline 25 & \begin{tabular}{|l|l|}
422 \\
\end{tabular} & 29 & 0.00060835 & 0.0024 & 410.9500 & 0.3547 & 27 & 0.00063636 & 0.0027 & 369.7528 & 0.3854 \\
\hline 26 & \begin{tabular}{|l|}
180 \\
\end{tabular} & 31 & 0.00053751 & 0.0027 & 372.0846 & 0.0265 & 28 & 0.00060006 & 0.0026 & 383.9882 & 0.0257 \\
\hline 27 & 10 & 35 & 0.00043850 & 0.0035 & 285.0602 & 0.9821 & 32 & 0.00047767 & 0.0035 & 281.7638 & 0.9829 \\
\hline 28 & 1146 & 30 & 0.00057648 & 0.0012 & 867.3393 & 0.4993 & 28 & 0.00059192 & 0.0015 & 670.3998 & 0.5914 \\
\hline 29 & \begin{tabular}{|l|}
600 \\
\end{tabular} & 31 & 0.00052915 & 0.0011 & 944.9138 & 0.0157 & 29 & 0.00053854 & 0.0014 & 711.4468 & 0.0209 \\
\hline 30 & 15 & 33 & 0.00046201 & 0.0014 & 721.4778 & 0.0487 & 30 & 0.00050901 & 0.0014 & 727.6242 & 0.0483 \\
\hline 31 & 36 & 35 & 0.00041217 & 0.0016 & 606.5403 & 0.0066 & 32 & 0.00044795 & 0.0017 & 589.0268 & 0.0068 \\
\hline 32 & 4 & 38 & 0.00035361 & 0.0021 & $\begin{array}{l}471.3229 \\
\end{array}$ & 0 & 34 & 0.00040303 & 0.0020 & 508.4395 & 0 \\
\hline 33 & 0 & 41 & 0.00031202 & 0.0025 & 400.6098 & 0.0198 & 38 & 0.00033081 & 0.0026 & 379.2781 & 0.0209 \\
\hline 34 & 8 & 46 & 0.00025893 & 0.0031 & 321.8382 & 0.5061 & 42 & 0.00028285 & 0.0031 & 319.6620 & 0.5084 \\
\hline 35 & 227 & 47 & 0.00025115 & 0.0030 & 331.8048 & 0.1779 & 43 & 0.00027335 & 0.0030 & 328.0981 & 0.1797 \\
\hline 36 & 65 & 52 & 0.00021546 & 0.0034 & 290.0747 & 0.4549 & 47 & 0.00023949 & 0.0034 & 293.2293 & 0.4513 \\
\hline 37 & 176 & 54 & 0.00020439 & 0.0035 & 287.8045 & 0.1825 & 49 & 0.00022567 & 0.0035 & 289.0592 & 0.1818 \\
\hline 38 & 58 & 60 & 0.00017613 & 0.0039 & $\begin{array}{l}258.0778 \\
\end{array}$ & 0.8298 & 55 & 0.00019135 & 0.0039 & 255.9210 & 0.8323 \\
\hline 39 & \begin{tabular}{|l|}
457 \\
\end{tabular} & 55 & 0.00019958 & 0.0032 & 313.1522 & 0.6163 & 50 & 0.00021970 & 0.0032 & 313.6941 & 0.6157 \\
\hline 40 & \begin{tabular}{|l|}
300 \\
\end{tabular} & 56 & 0.00019362 & 0.0031 & 322.7922 & 0.2596 & 51 & 0.00021248 & 0.0031 & 322.3448 & 0.2599 \\
\hline 41 & 97 & 60 & 0.00017518 & 0.0033 & 300.4454 & 0.5833 & 54 & 0.00019608 & 0.0033 & 305.5672 & 0.5771 \\
\hline 42 & \begin{tabular}{|l|}
263 \\
\end{tabular} & 61 & 0.00017088 & 0.0032 & 308 & 0.7695 & 55 & 0.00019071 & 0.0032 & 312.4837 & 0.7646 \\
\hline 43 & \begin{tabular}{|l|}
452 \\
\end{tabular} & 58 & 0.00018471 & 0.0028 & 360.9240 & 0.5066 & 53 & 0.00020147 & 0.0028 & 357.8521 & 0.5096 \\
\hline 44 & \begin{tabular}{|l|}
255 \\
\end{tabular} & 60 & 0.00017494 & 0.0028 & 357.2656 & 0.4239 & 55 & 0.00018977 & 0.0028 & 352.2385 & 0.4284 \\
\hline 45 & \begin{tabular}{|l|}
197 \\
\end{tabular} & 62 & 0.00016669 & 0.0028 & 352.8824 & 0.4213 & 57 & 0.00017993 & 0.0029 & 346.2687 & 0.4273 \\
\hline 46 & 193 & 65 & 0.00015501 & 0.0029 & 339.5275 & 0.0175 & 59 & 0.00017109 & 0.0029 & 341.0078 & 0.0174 \\
\hline 47 & 6 & 71 & 0.00013666 & 0.0033 & 304.8927 & 0.2283 & 65 & 0.00014872 & 0.0033 & 302.4713 & 0.2299 \\
\hline 48 & 79 & 77 & 0.00012203 & 0.0035 & 282.5769 & 0.9443 & 70 & 0.00013431 & 0.0035 & 282.8895 & 0.9441 \\
\hline 49 & 816 & 67 & 0.00014865 & 0.0027 & 373.7313 & 0.9731 & 61 & 0.00016321 & 0.0027 & 373.3741 & 0.9732 \\
\hline 50 & 1351 & 59 & 0.00018299 & 0.0016 & 607.1933 & 0.2163 & 54 & 0.00019859 & 0.0017 & 592.4077 & 0.2211 \\
\hline 51 & \begin{tabular}{|l|}
148 \\
\end{tabular} & 61 & 0.00017286 & 0.0017 & $\begin{array}{l}578.5157 \\
\end{array}$ & 0.0356 & 56 & 0.00018647 & 0.0018 & 559.2125 & 0.0369 \\
\hline 52 & 21 & 65 & 0.00015463 & 0.0020 & 497.4630 & 0.3740 & 59 & 0.00017078 & 0.0020 & 501.3182 & 0.3717 \\
\hline 53 & 233 & 67 & 0.00014710 & 0.0021 & 485.5741 & 0.2412 & 61 & 0.00016149 & 0.0021 & 484.8984 & 0.2414 \\
\hline 54 & \begin{tabular}{|l|}
134 \\
\end{tabular} & 70 & 0.00013704 & 0.0022 & 456.0729 & 0.5429 & 64 & 0.00014927 & 0.0022 & 450.8378 & 0.5470 \\
\hline 55 & \begin{tabular}{|l|}
357 \\
\end{tabular} & 71 & 0.00013390 & 0.0021 & 466.7511 & 0.3387 & 65 & 0.00014548 & 0.0022 & 459.7839 & 0.3428 \\
\hline 56 & 193 & 74 & 0.00012518 & 0.0023 & 443.8036 & 0.4124 & 67 & 0.00013886 & 0.0022 & 448.9809 & 0.4088 \\
\hline 57 & 236 & 76 & 0.00012015 & 0.0023 & 438.0646 & 0.0683 & 69 & 0.00013259 & 0.0023 & 440.2864 & 0.0680 \\
\hline 58 & 31 & 81 & 0.00010898 & 0.0025 & 398.9678 & 0.6034 & 73 & 0.00012180 & 0.0025 & 406.0499 & 0.5970 \\
\hline 59 & 369 & 81 & 0.00010912 & 0.0024 & 416.5716 & 0.8340 & 74 & 0.00011908 & 0.0024 & 413.4820 & 0.8362 \\
\hline 60 & 748 & 78 & 0.00011550 & 0.0021 & 481.0083 & 0 & 71 & 0.00012685 & 0.0021 & 480.6770 & 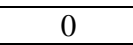 \\
\hline
\end{tabular}




\begin{tabular}{|c|c|c|c|c|c|c|c|c|c|c|c|}
\hline 61 & 0 & 82 & 0.00010707 & 0.0022 & 444.7502 & 0.4065 & 75 & 0.00011659 & 0.0023 & 440.0930 & 0.4097 \\
\hline 62 & 232 & 85 & 0.00010115 & 0.0023 & 429.8527 & 0.5359 & 77 & 0.00011205 & 0.0023 & 433.6545 & 0.5328 \\
\hline 63 & 330 & 86 & 0.00009942 & 0.0023 & 437.3230 & 0.5660 & 78 & 0.00010990 & 0.0023 & 440.2042 & 0.5636 \\
\hline 64 & 365 & 87 & 0.00009763 & 0.0022 & 445.3546 & 0.9357 & 79 & 0.00010770 & 0.0022 & 447.2760 & 0.9349 \\
\hline 65 & 1222 & 81 & 0.00010917 & 0.0017 & 572.5192 & 0.6127 & 73 & 0.00012232 & 0.0017 & 590.2688 & 0.6014 \\
\hline 66 & 543 & 81 & 0.00010925 & 0.0016 & 610.2101 & 0.0163 & 74 & 0.00011910 & 0.0017 & 602.3228 & 0.0165 \\
\hline 67 & 10 & 85 & 0.00010071 & 0.0018 & 551.6600 & 0.0286 & 77 & 0.00011165 & 0.0018 & 558.7317 & 0.0282 \\
\hline 68 & 16 & 89 & 0.00009357 & 0.0020 & 508.8922 & 0.6464 & 81 & 0.00010280 & 0.0020 & 508.7435 & 0.6465 \\
\hline 69 & 429 & 89 & 0.00009352 & 0.0019 & 534.6428 & 0.5078 & 81 & 0.00010275 & 0.0019 & 534.4750 & 0.5079 \\
\hline 70 & 379 & 90 & 0.00009192 & 0.0018 & 543.9800 & 0.0777 & 82 & 0.00010077 & 0.0018 & 542.2709 & 0.0779 \\
\hline 71 & 44 & 94 & 0.00008581 & 0.0020 & 506.6552 & 0.2248 & 86 & 0.00009338 & 0.0020 & 500.6751 & 0.2271 \\
\hline 72 & 129 & 98 & 0.00008038 & 0.0021 & 478.5080 & 0.8160 & 89 & 0.00008865 & 0.0021 & 480.4346 & 0.8147 \\
\hline 73 & 810 & 95 & 0.00008436 & 0.0019 & 538.8064 & 0.4162 & 86 & 0.00009362 & 0.0018 & 545.7881 & 0.4122 \\
\hline 74 & 290 & 97 & 0.00008162 & 0.0019 & 532.6786 & 0.4306 & 88 & 0.00009021 & 0.0019 & 536.5569 & 0.4283 \\
\hline 75 & 300 & 99 & 0.00007903 & 0.0019 & 527.2417 & 0.6333 & 90 & 0.00008700 & 0.0019 & 528.4436 & 0.6325 \\
\hline 76 & 529 & 99 & 0.00007902 & 0.0018 & 550.1894 & 0.3999 & 90 & 0.00008700 & 0.0018 & 551.5263 & 0.3992 \\
\hline 77 & 281 & 101 & 0.00007659 & 0.0018 & 544.0097 & 0.2548 & 92 & 0.00008401 & 0.0018 & 542.7668 & 0.2553 \\
\hline 78 & 160 & 105 & 0.00007195 & 0.0019 & 514.7588 & 0.7998 & 95 & 0.00007990 & 0.0019 & 521.0477 & 0.7959 \\
\hline 79 & 828 & 102 & 0.00007540 & 0.0017 & 576.6483 & 0.8268 & 93 & 0.00008255 & 0.0017 & 573.8421 & 0.8283 \\
\hline 80 & 1011 & 99 & 0.00007926 & 0.0015 & 664.0276 & 0.4884 & 91 & 0.00008536 & 0.0016 & 643.6806 & 0.4991 \\
\hline 81 & 445 & 101 & 0.00007643 & 0.0015 & 654.1981 & 0.3639 & 92 & 0.00008382 & 0.0015 & 652.2555 & 0.3648 \\
\hline 82 & 296 & 103 & 0.00007399 & 0.0016 & 643.6336 & 0.9346 & \begin{tabular}{|l|}
94 \\
\end{tabular} & 0.00008084 & 0.0016 & 638.2868 & 0.9360 \\
\hline 83 & 1755 & 98 & 0.00008059 & 0.0012 & 827.2104 & 0.7237 & 89 & 0.00008896 & 0.0012 & 834.5513 & 0.7206 \\
\hline 84 & 1064 & 97 & 0.00008221 & 0.0011 & 935.6319 & 0.8513 & 88 & 0.00009098 & 0.0011 & 950.7763 & 0.8467 \\
\hline 85 & 1783 & 95 & 0.00008559 & 0.00085593 & 1168.3 & 0.5210 & 87 & 0.00009260 & 0.00089357 & 1119.1 & 0.5363 \\
\hline 86 & 860 & 96 & 0.00008362 & 0.00083622 & 1195.9 & 0.5604 & 87 & 0.00009285 & 0.00081149 & 1232.3 & 0.5496 \\
\hline 87 & 983 & 96 & 0.00008379 & 0.00075414 & 1326 & 0.4133 & 88 & 0.00009038 & 0.00079807 & 1253 & 0.4312 \\
\hline 88 & 707 & 97 & 0.00008201 & 0.00073805 & 1354.9 & 0.0241 & 89 & 0.00008824 & 0.00078709 & 1270.5 & 0.0256 \\
\hline 89 & 33 & 100 & 0.00007680 & 0.00084479 & 1183.7 & 0.5197 & 91 & 0.00008439 & 0.00084479 & 1183.7 & 0.5197 \\
\hline 90 & 868 & 100 & 0.00007703 & 0.00077027 & 1298.2 & 0.4275 & 91 & 0.00008464 & 0.00077027 & 1298.2 & 0.4275 \\
\hline 91 & 724 & 101 & 0.00007547 & 0.00075472 & 1325 & 0.8268 & 92 & 0.00008273 & 0.00076031 & 1315.3 & 0.8290 \\
\hline 92 & 2323 & 100 & 0.00007689 & 0.00061510 & 1625.8 & 0.8351 & 92 & 0.00008205 & 0.00067937 & 1472 & 0.8634 \\
\hline 93 & 2930 & 99 & 0.00007850 & 0.00047102 & 2123.1 & 0.4975 & 93 & 0.00007875 & 0.00065918 & 1517 & 0.6183 \\
\hline 94 & 1461 & 99 & 0.00007877 & 0.00039382 & 2539.2 & 0.2825 & 94 & 0.00007643 & 0.00064659 & 1546.6 & 0.4202 \\
\hline 95 & 843 & 101 & 0.00007463 & 0.00044779 & 2233.2 & 0.0054 & 95 & 0.00007453 & 0.00063722 & 1569.3 & 0.0076 \\
\hline 96 & 12 & 102 & 0.00007325 & 0.00043947 & 2275.4 & 0.1084 & 96 & 0.00007315 & 0.00063198 & 1582.3 & 0.1521 \\
\hline 97 & 261 & 104 & 0.00006988 & 0.00048917 & 2044.3 & 0.5854 & 97 & 0.00007171 & 0.00062606 & 1597.3 & 0.6760 \\
\hline 98 & 1800 & 104 & 0.00006997 & 0.00041980 & 2382.1 & 0.3045 & 98 & 0.00006960 & 0.00061388 & 1629 & 0.4120 \\
\hline 99 & 865 & 106 & 0.00006658 & 0.00046604 & 2145.7 & 0.4877 & 99 & 0.00006798 & 0.00060573 & 1650.9 & 0.5807 \\
\hline 100 & 1435 & 106 & 0.00006680 & 0.00040079 & 2495.1 & 0.0120 & 100 & 0.00006618 & 0.00059561 & 1678.9 & 0.0177 \\
\hline 101 & 30 & 108 & 0.00006387 & 0.00044710 & 2236.7 & 0.0619 & 101 & 0.00006502 & 0.00059103 & & 0.0810 \\
\hline 102 & 143 & 110 & 0.00006120 & 0.00048959 & 2042.5 & 0.0515 & 102 & 0.00006388 & 0.00058638 & 1705.4 & 0.0614 \\
\hline 103 & 108 & 112 & 0.00005879 & 0.00052906 & 1890.1 & 0 & 103 & 0.00006280 & 0.00058215 & 1717.8 & 0 \\
\hline 104 & 0 & 114 & 0.00005662 & 0.00056622 & 1766.1 & 0.8281 & 104 & 0.00006181 & 0.00057859 & 1728.3 & 0.8346 \\
\hline 105 & 3110 & 113 & 0.00005762 & 0.00046092 & 2169.6 & 0.4372 & 105 & 0.00005976 & 0.00056476 & 1770.7 & 0.5055 \\
\hline 106 & 1247 & 114 & 0.00005641 & 0.00045129 & 2215.9 & 0.3466 & 106 & 0.00005839 & 0.00055704 & 1795.2 & 0.4086 \\
\hline 107 & 943 & 115 & 0.00005532 & 0.00044256 & 2259.6 & 0.2664 & 107 & 0.00005716 & 0.00055044 & 1816.7 & 0.3198 \\
\hline 108 & 700 & 116 & 0.00005432 & 0.00043459 & 2301 & 0.3163 & 108 & 0.00005604 & 0.00054476 & 1835.7 & 0.3791 \\
\hline 109 & 875 & 118 & 0.00005207 & 0.00046861 & 2134 & 0.1085 & 109 & 0.00005492 & 0.00053877 & 1856.1 & 0.1237 \\
\hline 110 & 245 & 119 & 0.00005128 & 0.00046152 & 2166.7 & 0.2857 & 110 & 0.00005401 & 0.00053474 & 1870.1 & 0.3228 \\
\hline 111 & 729 & 121 & 0.00004929 & 0.00049291 & 2028.8 & 0.6074 & 111 & 0.00005301 & 0.00052959 & 1888.3 & 0.6338 \\
\hline 112 & 1897 & 121 & 0.00004932 & 0.00044388 & 2252.9 & 0.1800 & 112 & 0.00005173 & 0.00052149 & 1917.6 & 0.2079 \\
\hline 113 & 447 & 123 & 0.00004748 & 0.00047481 & 2106.1 & 0.1675 & 113 & 0.00005086 & 0.00051723 & 1933.4 & 0.1810 \\
\hline 114 & 386 & 124 & 0.00004679 & 0.00046786 & 2137.4 & 0.1883 & 114 & 0.00005003 & 0.00051330 & 1948.2 & 0.2046 \\
\hline 115 & 446 & 126 & 0.00004514 & 0.00049652 & 2014 & 0.0588 & 115 & 0.00004922 & 0.00050940 & 1963.1 & 0.0603 \\
\hline 116 & 122 & 128 & 0.00004368 & 0.00052411 & 1908 & 0.4048 & 117 & 0.00004745 & 0.00054282 & 1842.2 & 0.4157 \\
\hline 117 & 990 & 129 & 0.00004298 & 0.00051575 & 1938.9 & 0.3867 & 118 & 0.00004660 & 0.00053735 & 1861 & 0.3992 \\
\hline 118 & 948 & 131 & 0.00004148 & 0.00053930 & 1854.2 & 0.4421 & 119 & 0.00004579 & 0.00053212 & 1879.3 & 0.4377 \\
\hline 119 & 1082 & 132 & 0.00004082 & 0.00053072 & 1884.2 & 0.0116 & 120 & 0.00004498 & 0.00052668 & 1898.7 & 0.0115 \\
\hline 120 & 22 & 134 & 0.00003963 & 0.00055479 & 1802.5 & 0.0408 & 122 & 0.00004349 & 0.00055672 & 1796.2 & 0.0409 \\
\hline 121 & 75 & 136 & 0.00003851 & 0.00057759 & 1731.3 & 0.2430 & 124 & 0.00004211 & 0.00058497 & 1709.5 & 0.2457 \\
\hline 122 & 482 & 138 & 0.00003738 & 0.00059810 & 1672 & 0.9629 & 126 & 0.00004075 & 0.00061043 & 1638.2 & 0.9654 \\
\hline 123 & 5509 & 134 & 0.00003966 & 0.00043622 & 2292.4 & 0.0427 & 123 & 0.00004258 & 0.00047135 & 2121.6 & 0.0460 \\
\hline 124 & 100 & 136 & 0.00003841 & 0.00046096 & 2169.4 & 0.0046 & 124 & 0.00004200 & 0.00046870 & 2133.6 & 0.0047 \\
\hline
\end{tabular}




\begin{tabular}{|c|c|c|c|c|c|c|c|c|c|c|c|}
\hline 125 & 10 & 138 & 0.00003727 & 0.00048457 & 2063.7 & 0.4049 & 126 & 0.00004061 & 0.00049749 & 2010.1 & 0.4130 \\
\hline 126 & 1071 & 139 & 0.00003672 & 0.00047740 & 2094.7 & 0.1623 & 127 & 0.00003994 & 0.00049285 & 2029 & 0.1671 \\
\hline 127 & 371 & 141 & 0.00003563 & 0.00049886 & 2004.6 & 0.3257 & 129 & 0.00003863 & 0.00051880 & 1927.5 & 0.3363 \\
\hline 128 & 790 & 143 & 0.00003455 & 0.00051822 & 1929.7 & 0.9587 & 130 & 0.00003806 & 0.00051457 & 1943.4 & 0.9578 \\
\hline 129 & 6150 & 139 & 0.00003672 & 0.00036720 & 2723.3 & 0.7046 & 129 & 0.00003822 & 0.00044373 & 2253.6 & 0.7709 \\
\hline 130 & 3321 & 139 & 0.00003666 & 0.00032992 & 3031 & 0.2916 & 130 & 0.00003727 & 0.00043605 & 2293.3 & 0.3660 \\
\hline 131 & 1045 & 140 & 0.00003608 & 0.00032470 & 3079.8 & 0.1897 & 131 & 0.00003664 & 0.00043193 & 2315.2 & 0.2441 \\
\hline 132 & 648 & 141 & 0.00003555 & 0.00031996 & 3125.4 & 0.8271 & 132 & 0.00003607 & 0.00042852 & 2333.6 & 0.9047 \\
\hline 133 & 5485 & 140 & 0.00003613 & 0.00025293 & 3953.6 & 0.2543 & 133 & 0.00003494 & 0.00041827 & 2390.8 & 0.3844 \\
\hline 134 & 1160 & 141 & 0.00003553 & 0.00024870 & 4020.9 & 0.3710 & 134 & 0.00003433 & 0.00041408 & 2415 & 0.5378 \\
\hline 135 & 1864 & 142 & 0.00003489 & 0.00024423 & 4094.4 & 0.6341 & 135 & 0.00003367 & 0.00040906 & 2444.6 & 0.8143 \\
\hline 136 & 4116 & 142 & 0.00003489 & 0.00020934 & 4777 & & 136 & 0.00003278 & 0.00040126 & 2492.1 & \\
\hline
\end{tabular}

Since the relationship between the reliability function $R\left(t_{i}\right)$ and the failure distribution function $F_{i}\left(t_{i}\right)$ are related by $R\left(t_{i}\right)=1-F_{i}\left(t_{i}\right)$, the quality of reliability prediction of the models may be judged by examining the estimated failure distribution functions of the models.

From Table 1, we can see that normally the failure rate for our modified J-M model with imperfect debugging is greater than or equal to the failure rate of the J-M model. It is also noted that the MTTF is infinite for some intervals of times between failures in J-M model. So this model assumes that there are no more faults in the software although faults are present in the software at that time. In our modified J-M model, the MTTF is not infinite for every interval between failures. So this is more realistic in practical sense as it implies that till then fault is remaining in the software. This is the most practical situation in software fault debugging process. When $p \rightarrow 1$ and $r \rightarrow 0$ i.e. for perfectly debugging procedure, our considered imperfect debugging modified J-M model approaches to the J-M model.

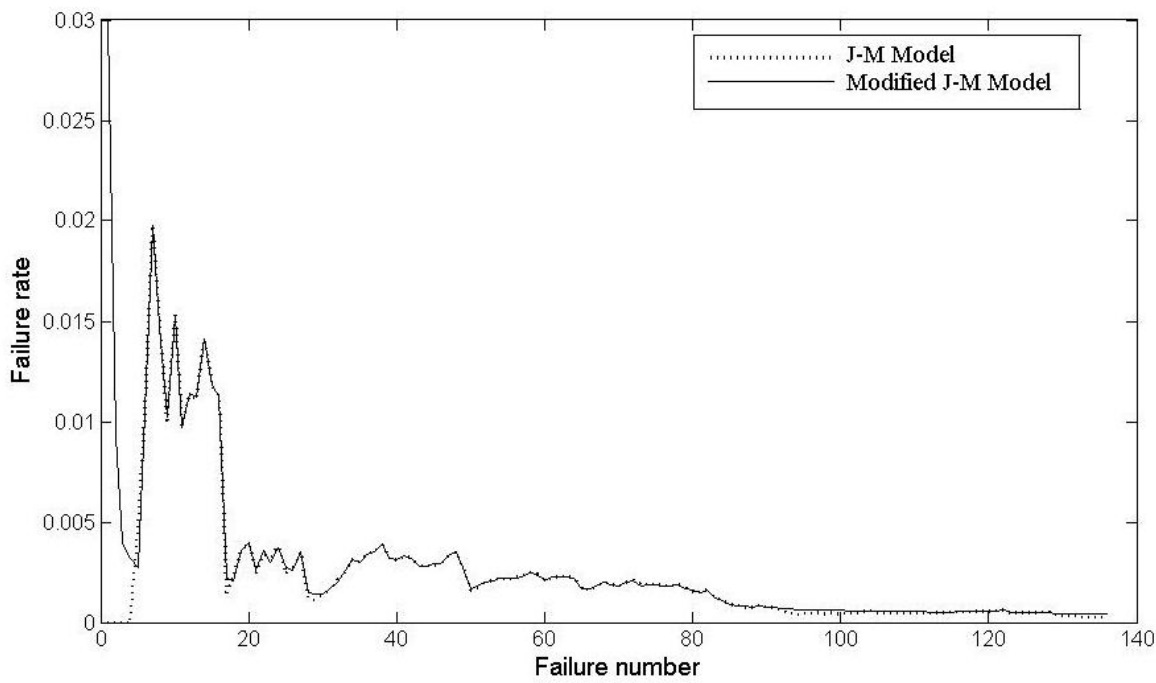

Fig 1: Failure rate as a function of failure number

A plot of failure rate against failure number for the J-M model and the modified J-M model is shown in Figure 1. From the graph of Figure 1, we get the clear idea about the failure behavior of the two models. The failure rate is maximum at the beginning of the testing process. As the fault content of the software is decreasing i.e. the failure number is increasing, the failure rate of our model is decreasing. This practical event occurs at the time of debugging.

\subsubsection{Prediction analysis}

In order to compare the quality of prediction of the two models, we consider the sequences of probabilities for $T_{i}=t_{i}$. Let $u_{i}=\hat{F}_{i}\left(t_{i}\right)$. If each of the estimated $\hat{F}_{i}$ is equal to the true $F_{i}$, the sequence $\left\{u_{i}\right\}$ should look like a realization of independent uniform $U(0,1)$ random variables [19,20,21]. We can examine the quality of each model by plotting the sample distribution function of the $u_{i}$ 's and comparing it with the distribution function of $U(0,1)$, which is the line of unity slope through the origin. We use the quantile-quantile plot [4] which is a plot of the order set of $n$ number of $u_{i}$ 's against $i / n$, where $n$ is the sample size and $i$ is the rank of the sample point. The closeness of the plot to the line of unity slope is an indication of the closeness of the $u_{i}$ 's to uniformity and so an indication of the quality of prediction of the model. The closer the plot to the line of unity slope, the more likely the $u_{i}$ 's is from a random sample of the uniform distribution, and so the better the model. The goodness-of-fit test can be performed by formal statistical tests. In this paper, we have used the Kolmogorov-Smirnov (KS) test. KS distance is the maximum vertical deviation between the plot and the line of unity slope. 


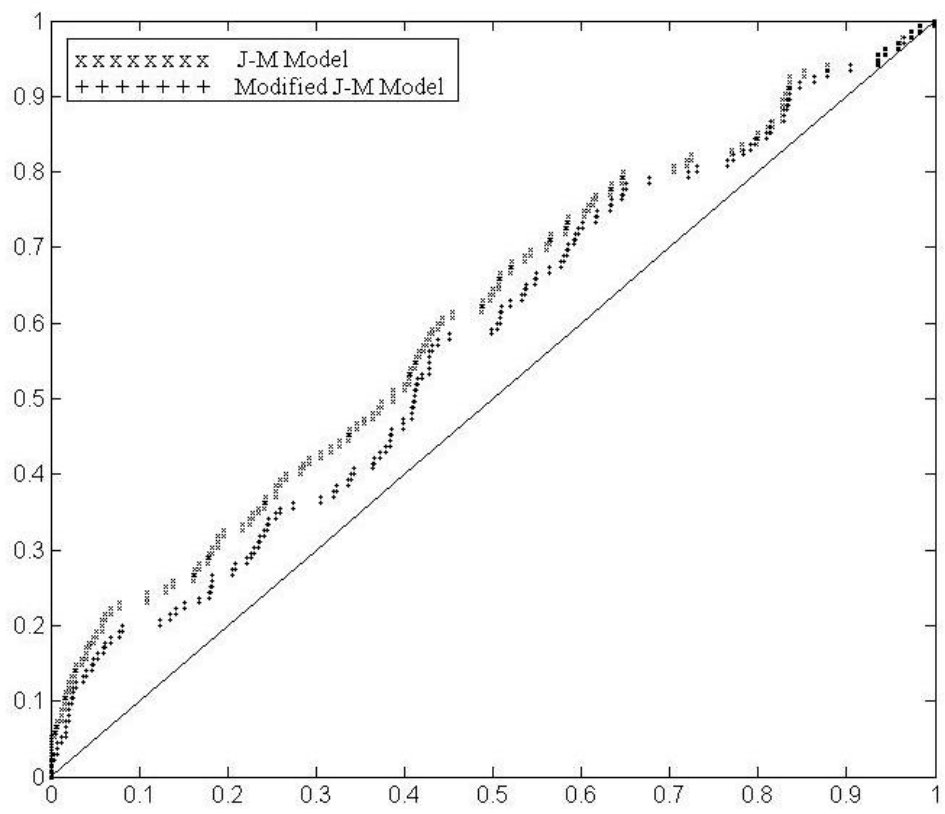

Fig 2: Quantile-quantile plot of the data in Table 1

The quantile-quantile plot of the data in Table 1 is shown in Figure 2. The KS distance is 0.165 for the J-M model, and 0.14 for the modified model. The results show that the modified model yields a better prediction in this case. constant. First, we consider the fixed value of $q$ at 0.05 and the values of $p$ and $r$ are taken as follows:

$p=0.94$ and $r=0.01 ; p=0.92$ and $r=0.03 ; p=0.9$ and $r=0.05$

\subsection{Sensitivity Analysis of the Proposed}

\section{Model}

To study the performance of our modified J-M model with imperfect debugging, sensitivity analyses have been presented graphically on the different values of $p$ and $r$ keeping $q$ as

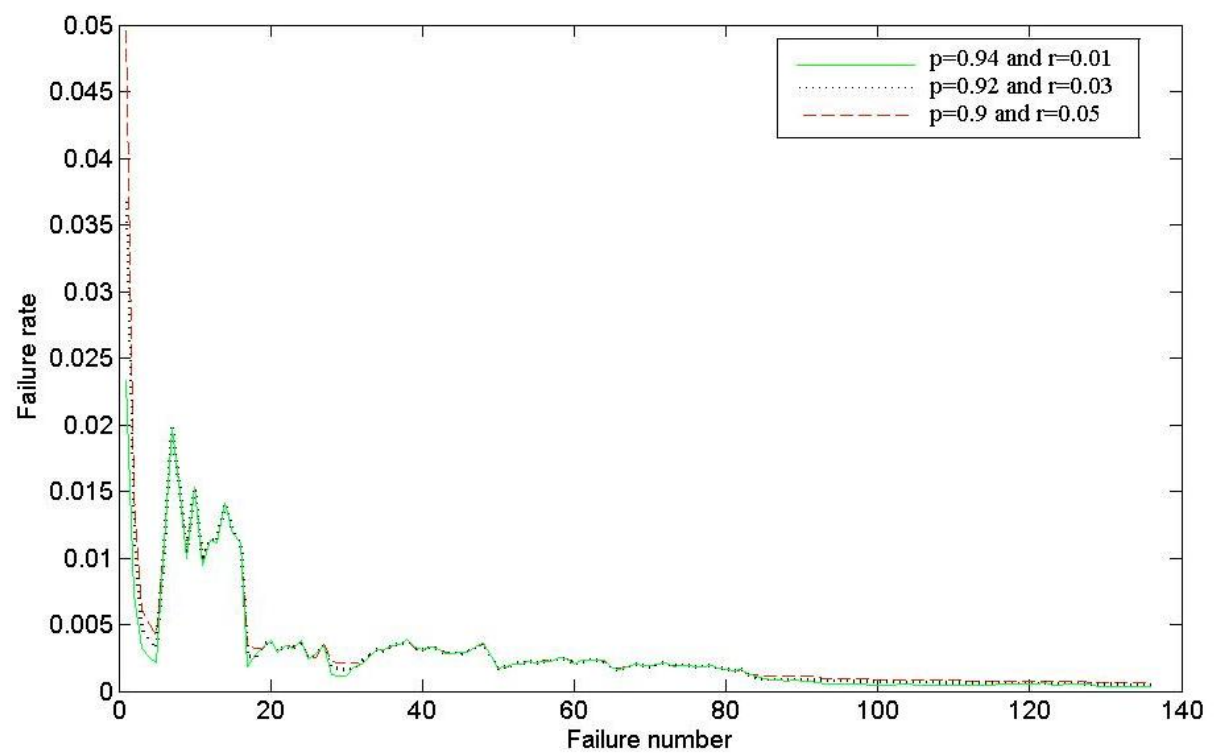

Fig 3: Failure rate against failure number for different values of $p$ and $r$

A plot of failure rate versus failure number for the above different values of $p$ and $r$ of the modified J-M model is shown in Figure 3. From the graph, we can see that the failure rate is maximum for $p=0.9$ and $r=0.05$. But as $\mathrm{p}$ is increasing and $r$ is decreasing, we are able to remove more and more faults perfectly. So the failure rate is decreasing which can be found from the Figure 3 . 


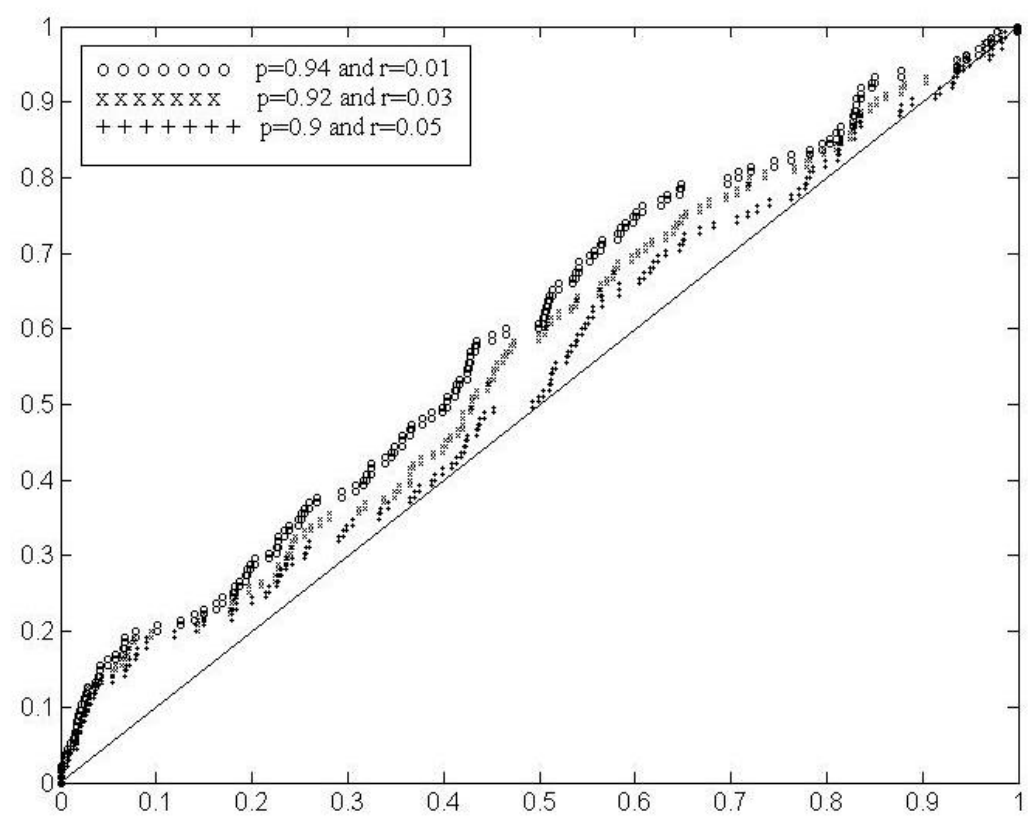

Fig 4: Quantile-quantile plots for different values of $p$ and $r$

Quantile-quantile plots for the different values of $p$ and $r$ on the fixed value of $\mathrm{q}$ at 0.05 of our modified J-M model are shown in Figure 4.
Now we show the KS distances for the different values of $q$ keeping it constant for the different values of $\mathrm{p}$ and $\mathrm{r}$ in Table 2.

Table 2. KS distances for different values of $p$ and $\mathbf{r}$ for different value of $q$

\begin{tabular}{|c|c|c|c|c|c|c|c|c|}
\hline \multicolumn{3}{|c|}{ For $\mathrm{q}=0.07$} & \multicolumn{3}{c|}{ For $\mathrm{q}=0.05$} & \multicolumn{3}{|c|}{ For q=0.04 } \\
\hline $\mathrm{p}$ & $\mathrm{R}$ & KS distance & $\mathrm{p}$ & $\mathrm{r}$ & KS distance & $\mathrm{p}$ & $\mathrm{r}$ & KS distance \\
\hline 0.92 & 0.01 & 0.140078 & 0.94 & 0.01 & 0.155263 & 0.95 & 0.01 & 0.161781 \\
\hline 0.9 & 0.03 & 0.110993 & 0.92 & 0.03 & 0.115093 & 0.94 & 0.02 & 0.145678 \\
\hline 0.88 & 0.05 & 0.093693 & 0.9 & 0.05 & 0.101993 & 0.93 & 0.03 & 0.129978 \\
\hline
\end{tabular}

From Table 2, we can see that for fixed value of $q$, as $\mathrm{p}$ is decreasing and $r$ is increasing, the KS distance is decreasing. So we get better predictions as $\mathrm{p}$ is decreasing and $\mathrm{r}$ is increasing for some fixed value of q. Again, for fixed value of $r$, as $p$ is increasing and $q$ is decreasing, the KS distance is increasing. It is also noted that for fixed value of $p$, as $q$ is decreasing and $\mathrm{r}$ is increasing, the KS distance is decreasing. So in this case we also get better predictions. From Table 2, it is clear that we get better predictions in our model for the smaller value of (p-r). In reality, the value of (p-r) is less than 1 and it decreases in most of the cases of debugging process during testing. So our model excellently predicts the software reliability in practical sense.

\section{CONCLUSION}

We develop a modified J-M model which assumes the imperfect debugging process in fault removal activity during the testing phase. In our proposed model, we assume that whenever a failure occurs, the detected fault is not perfectly removed and there is a chance of raising new fault/s. We consider that the perfect debugging probability, the imperfect debugging probability and the probability of arising new fault are independent of the testing time. A set of failure data is given for illustration. From the experimental results, we have seen that the failure rate is normally high for our modified $\mathrm{J}$ $\mathrm{M}$ model. So we need to increase the probability of perfect debugging and to decrease the probability of raising new fault/s during the fault removing process. Again, the difference between the probability of perfect debugging and the probability of raising new fault/s should be decreased to get better software reliability prediction. The experimental results also show that our proposed model yields a better prediction than the J-M model.

\section{ACKNOWLEDGEMENTS}

The authors wish to acknowledge the financial support given to this work through the research project (No. 25(0191)/10/EMR-II) by the Council of Scientific and Industrial Research, New Delhi, India.

\section{REFERENCES}

[1] Lyu, M.R. 1996. Handbook of Software Reliability Engineering. McGraw-Hill.

[2] Musa, J.D., Iannino, A., and Okumoto, K. 1990. Software Reliability: Measurement, Prediction, Application. McGraw-Hill.

[3] Jelinski, Z. and Moranda, P.B. 1972. Software reliability research, Statistical Computer Performance Evaluation. Academic Press: New York, 465-484. 
[4] Littlewood, B. 1987. How good are software reliability predictions?. Software Reliability: Achievement and Assessment. Blackwell Scientific Publications. 154-166.

[5] Musa J.D. 1975. A theory of software reliability and its application. IEEE T. Software Eng. 1(3), 312-327.

[6] Goel, A.L., and Okumoto, K. 1979. Time dependent error detection rate model for software reliability and other performance measures. IEEE T. Reliab. R-28(3), 206-211.

[7] Yamada, S., Ohba, M. and Osaki, S. 1983. S-shaped reliability growth modeling for software error detection. IEEE T. Reliab. R-32(5), 475-484.

[8] Ohba, M. 1984. Software reliability analysis models. IBM J. Res. Dev. 28(4), 428-443.

[9] Goel, A.L. 1985. Software reliability models: assumptions, limitations and applicability. IEEE $\mathrm{T}$. Software Eng. SE-11(12), 1411-1423.

[10] Kapur, P.K., and Garg, R.B. 1990. Optimal release policy for software reliability growth models under imperfect debugging. Oper. Res. RAIRO. 24(3), 295305.

[11] Chang, Y.C., and Liu, C.T. 2009. A generalized JM model with applications to imperfect debugging in software reliability. Appl. Math. Model. 33, 3578-3588.
[12] Shyur, H.J. 2003. A stochastic software reliability model with imperfect-debugging and change-point. J. Syst. Software. 66(2), 135-141.

[13] Kapur, P.K., Singh, O.M.P., Shatnawi, O., and Gupta, A. 2006. A discrete NHPP model for software reliability growth with imperfect fault debugging and fault generation. Int. J. Perform. Eng. 2(4), 351-368.

[14] Prasad, R.S., Raju, O.N., and Kantam, R.R.L. 2010. SRGM with imperfect debugging by genetic algorithms. Int. J. Software Eng. Appl. 1(2), 66-79.

[15] Raju, O.N. 2011. Software reliability growth models for the safety critical software with imperfect debugging. Int. J. Comput. Sci. Eng. 3(8), 3019-3026.

[16] Xie, M. Dai, Y.S. and Poh, K.L. 2004. Computing System Reliability Models and Analysis. Kluwer Academic Publisher.

[17] Kremer, W. 1983. Birth-death and bug counting. IEEE T. Reliab. R-32(1), 37-47.

[18] Musa, J.D. 1980. Software Reliability Data. Data \& Analysis Center for Software.

[19] Dawid, A.P. 1984. Statistical theory: the prequential approach. J. Roy. Stat. Soc. A. 147, 278-292.

[20] Pham, H. 2006. System Software Reliability. Springer.

[21] Bittanti, S. 1988. Software Reliability Modelling and Identification (Lecture Notes in Computer Science). Springer-Verlag. 